Rabaska

Revue d'ethnologie de l'Amérique française

CSERGo, JULIA. La Gastronomie est-elle une marchandise culturelle comme une autre ? La gastronomie française à l'UNESCO : histoire et enjeux. Chartres, Menu Fretin, 2016, 313 p. ISBN 9791096339037

\title{
Myriam Mathieu-Bédard
}

Volume 15, 2017

URI : https://id.erudit.org/iderudit/1041142ar

DOI : https://doi.org/10.7202/1041142ar

Aller au sommaire du numéro

Éditeur(s)

Société québécoise d'ethnologie

ISSN

1703-7433 (imprimé)

1916-7350 (numérique)

Découvrir la revue

Citer ce compte rendu

Mathieu-Bédard, M. (2017). Compte rendu de [CSERGo, JuliA. La Gastronomie est-elle une marchandise culturelle comme une autre ? La gastronomie française à l'UNESCO : histoire et enjeux. Chartres, Menu Fretin, 2016, 313 p.

ISBN 9791096339037]. Rabaska, 15, 238-242. https://doi.org/10.7202/1041142ar

Ce document est protégé par la loi sur le droit d'auteur. L'utilisation des services d'Érudit (y compris la reproduction) est assujettie à sa politique d'utilisation que vous pouvez consulter en ligne.

https://apropos.erudit.org/fr/usagers/politique-dutilisation/ 
représentation du Christ, la partie centrale est réservée pour Dieu tandis que les ailes sont dédiées aux saints.

La restauration révèle l'importance des essences de bois et des techniques de production. Elle confirme que les meubles ont été modifiés au fil des années et qu'ils ont tous été redorés ou repeints. Les vastes églises du $\mathrm{XIX}^{\mathrm{e}}$ siècle ont amené les sculpteurs à agrandir les tabernacles ou à modifier celui qui était en place pour l'équilibrer dans son espace. Souvent, on ajoutait un gradin pour élever le tabernacle. Enfin, le nombre accru de fidèles a obligé la production de vases sacrés plus grands et l'espace eucharistique fut à son tour agrandi.

Cette étude magistrale présente plus de 200 photographies, dont la majorité est en couleur et inédite. Elles permettent d'appréhender en entier les tabernacles et des détails révélant la richesse de la décoration. En annexe, les auteurs ont réuni en un répertoire photographique des tabernacles étudiés. Les historiens étudieront avec plaisir une carte générale - déployée sur $120 \mathrm{~cm}$ présentant les paroisses et les missions le long du fleuve Saint-Laurent, de Rimouski à Côteau-des-Cèdres vers 1740. Le format, agréable pour la lecture, est néanmoins peu pratique pour ceux et celles qui voudraient partir à la découverte in situ de ces œuvres. Il en est de même pour le répertoire dont la consultation est difficile. Une version détachée aurait facilité la lecture.

Cette collaboration entre l'histoire de l'art et la restauration produit une étude originale, complète et exemplaire. C'est une contribution majeure à l'histoire de la sculpture québécoise d'origine et un ouvrage de référence essentiel pour les chercheurs et les amateurs d'art religieux ancien. Avec les auteurs, nous souhaitons l'élaboration d'une étude similaire pour les tabernacles du XIX ${ }^{\mathrm{e}}$ siècle.

Diane Joly Société québécoise d'ethnologie

CSERgo, Julia. La Gastronomie est-elle une marchandise culturelle comme une autre ? La gastronomie française à l'UNESCO : histoire et enjeux. Chartres, Menu Fretin, 2016, 313 p. ISBN 9791096339037.

L'une des conséquences de la Convention pour la sauvegarde du patrimoine culturel immatériel (PCI), adoptée par l'UNESCO en 2003, a été de faire de ce type de patrimoine un enjeu politique et économique sur les scènes nationales et internationale. Et c'est en envisageant cette Convention comme outil de gouvernance que l'historienne Julia Csergo, responsable scientifique de la candidature du « repas gastronomique des Français » à l'UNESCO, 
ajouté en 2010 à la Liste représentative du patrimoine culturel immatériel de l'humanité, conclut que cette inscription semble avoir peu bénéficié au peuple français et à ses professionnels de la gastronomie. En effet, la France aurait, notamment «par manque de volonté politique, [...] raté la cible» (p. 13) en n'utilisant pas - comme d'autres pays l'ont fait - la Convention pour défendre sa gastronomie au moyen d'une politique culturelle et économique, et ce, au nom de la sauvegarde de la diversité culturelle que menace la mondialisation. Avec cet ouvrage, Csergo présente un bilan critique de l'inscription controversée de la gastronomie française à l'UNESCO. Elle revient sur les buts de cette candidature, sur les débats qu'elle a suscités ainsi que sur les utilisations faites de l'inscription jusqu'ici. Elle formule aussi des propositions sur ce qu'il conviendrait dorénavant de faire selon elle. L'auteure annonce deux objectifs à cette publication hautement critique qui veut enjoindre à prendre les moyens nécessaires pour protéger les produits et les savoir-faire de la gastronomie française. En relatant l'évolution et les écueils du processus d'inscription et de ses suites, Csergo souhaite d'abord tirer une leçon du passé pour mieux envisager l'avenir. Elle désire aussi continuer son engagement en tant que scientifique en présentant de nouvelles propositions et une « analyse réflexive » (p. 19) de sa propre démarche, libérée de l'autocensure et du devoir de réserve que la nature fortement politique du projet a pu lui imposer auparavant.

Dans une approche qui se situe " entre micro-histoire et égo-histoire » (p. 19), l'auteure dresse, avec patience et efficacité, le portrait du cas d'étude, à l'aide notamment d'éléments chronologiques et contextuels. Ce faisant, elle identifie, tant avant qu'après l'inscription de 2010, les pierres d'achoppement de ce dossier complexe qui souligne bien certaines des ramifications politiques et économiques de la Convention de 2003.

Didactique, l'essai est organisé de manière quasi circulaire autour de la question évoquée dans le titre. L'auteure présente dès le départ ses principaux constats et arguments, dont elle expose ensuite les fondements au fil des chapitres. Elle annonce d'entrée de jeu que, puisqu'on a officiellement reconnu certains éléments gastronomiques comme patrimoniaux - ce qui signifie qu'ils sont des objets à contenu culturel - et que ceux-ci renvoient à des biens et services, la gastronomie devrait être reconnue en tant que domaine culturel et marchandise culturelle comme les autres. À ce titre, elle devrait bénéficier d'une politique culturelle visant à protéger ses produits et ses acteurs, menacés par l'uniformisation qu'engendre la mondialisation. Selon Csergo, une telle politique aurait pu résulter de l'inscription, si le dossier avait été mené avec une meilleure compréhension des enjeux de la Convention et avec la volonté politique nécessaire. 
Csergo déplore aussi d'emblée que la version définitive de la candidature ait décrit le repas gastronomique des Français comme une pratique sociale sans l'ancrer dans une matérialité, c'est-à-dire sans faire référence à un produit alimentaire (ou à des producteurs), bien que la Convention souligne que le $\mathrm{P}_{\mathrm{CI}}$ est aussi associé à des dimensions matérielles. Par cette exclusion, qu'il est le seul à présenter parmi les éléments alimentaires inscrits à l'UNESCO, le dossier français ignorerait certaines directives opérationnelles qui encouragent les États parties à la Convention à utiliser le PCI comme « force motrice du développement économique inclusif et équitable » (art. 184, cité à la p. 25) et à considérer son potentiel pour le développement du tourisme durable. En effet, l'absence de produits ou de producteurs dans la candidature les exclut du plan de sauvegarde qui doit accompagner le dossier. C'est là, selon Csergo, une occasion manquée pour la France, qui ignorerait ainsi, à la différence d'autres pays, un rôle important que peut jouer la Convention, celle-ci « s'intégr[ant...] dans un dispositif d'instruments internationaux relatifs à la sauvegarde de la diversité culturelle [...] qui peuvent protéger des règles de marchés, les biens et services culturels inscrits dans le territoire des identités » (p. 13).

L'histoire critique de l'auteure suit ces constats. Bien documentée, elle aborde plusieurs questions et enjeux complexes qui ont influencé à la fois la candidature de la " gastronomie française » à l'UNESCO, souhaitée par le président Sarkozy, et la situation actuelle que l'auteure déplore. Qu'est-ce que la gastronomie ${ }^{3}$ ? Comment une certaine culture de la table est-elle devenue en France un fondement de la culture et de l'identité nationales, un patrimoine commun ? Comment la gastronomie, étroitement liée à l'économie et à la politique depuis des siècles, a-t-elle été utilisée, de manière plus ou moins heureuse, par différents régimes et hommes politiques français jusqu'à nos jours ? Comment le domaine alimentaire et gastronomique s'impose-t-il graduellement, malgré des réticences initiales de l'UNESCO, comme un domaine culturel concerné par la Convention de 2003 ? Pourquoi la France n'a-t-elle pas assumé un rôle déterminant dans la constitution de ce domaine culturel? Comment le dossier de candidature, d'abord évoqué comme celui de la «gastronomie française », a-t-il été élaboré autour du « repas gastronomique des Français » ? Pourquoi a-t-il finalement été « vid[é] d'une grande partie de son contenu » (p. 217) initial?

Après avoir fait le point sur le déficit de gouvernance associé au plan de sauvegarde, qui aurait occasionné des initiatives désorganisées, souvent

3. L'absence de consensus sur la définition de la gastronomie a créé une ambiguité autour de la candidature française. La définition anglo-saxonne du mot fait référence à la haute cuisine et aux grands chefs - à une culture élitiste et exclusive - tandis qu'une autre définition réfère plutôt à une « culture de la qualité et du plaisir de la table» (p. 93), qui serait constitutive de la culture et de l'identité françaises. 
peu efficaces et parfois contraires à l'esprit de la Convention et aux engagements de la France, l'essai se conclut sur des recommandations qui visent à défendre la gastronomie française de façon appropriée. Selon Csergo, la France devrait, entre autres, se munir d'une législation adéquate sur le $\mathrm{P}_{\mathrm{CI}}$ qui fasse du statut patrimonial une véritable protection pour les cultures immatérielles, « imposer le sens, historique et français, du terme gastronomie » (p. 292), puis reconnaître la gastronomie comme domaine culturel et la faire bénéficier, on le répète, d'une politique culturelle.

Bien que l'inscription du repas gastronomique des Français ait déjà fait l'objet d'autres publications (notamment de Csergo), la liberté que se donne ici l'auteure, sa posture particulièrement critique, son regard tourné vers l'avenir ainsi que son appel à l'action font l'originalité de cet essai. Mené de façon efficace et bénéficiant de la position privilégiée de son auteure dans le dossier, l'ouvrage est convaincant dans son ensemble.

On aurait cependant souhaité que Csergo, dont l'argumentation repose sur le levier que peut représenter la Convention pour « l'économie de la culture immatérielle » (p. 23), aille au-delà d'une description assez générale des gestes que d'autres pays ont annoncés pour soutenir les biens et services qui assurent la transmission des éléments alimentaires qu'ils ont fait inscrire à l'UNESCO (notamment afin d'en faire des pôles d'attraction touristique ou d'en favoriser la production et le commerce), ce qu'elle aurait pu faire en s'attardant par exemple (et si possible) à l'efficacité de ces mesures. De plus, toujours parce qu'elle envisage la Convention comme un outil permettant la protection des éléments inscrits par la mise en place de mesures économiques, il semble qu'il aurait été pertinent que l'auteure discute des mises en garde de l'UNESCO contre les effets parfois dénaturants de la valorisation commerciale et touristique du $\mathrm{PCI}^{4}$. On peut aussi relever que l'essai, destiné principalement à un auditoire français, pourrait parfois être déroutant pour un lecteur peu familier avec l'appareil gouvernemental de la France. Au niveau de la forme, en plus d'un certain nombre de coquilles, on remarque quelques tics du langage - les pages 30 et 31 présentent par exemple sept fois le mot « c'est-à-dire »- qu'un éditeur plus vigilant aurait pu corriger. Malgré ces quelques remarques, le livre demeure intéressant. En effet, au-delà de son plaidoyer en faveur de l'instauration d'une politique culturelle de la gastronomie en France, l'ouvrage apparaît pertinent, ne serait-ce que pour le regard critique qu'il pose sur la nature éminemment politique des inscriptions à l'UNESCO et sur l'action (ou l'inaction) des gouvernements en ce qui a trait à la protection de la culture et du patrimoine immatériel. La sonnette

4. Elle aborde la directive opérationnelle qui met en garde contre le détournement commercial, mais pas celle qui concerne les effets que les activités commerciales pourraient avoir sur la viabilité ou la nature même du PcI. 
d'alarme que veut être cet essai pourrait ainsi revêtir un intérêt particulier pour le lecteur québécois dans le contexte du renouvellement de la politique culturelle du Québec.

MYriam MathiEU-BÉdaRd Université du Québec à Montréal

Demester, Vincent. La Cuisine des premiers migrants du Québec. Enquête sur la disparition du patrimoine culinaire du Poitou-Charentes. Préface de Michel Suire et Pauline Arsenault. Paris, L'Harmattan, « Questions alimentaires et gastronomiques », 2014, 203 p. ISBN 978-2-343-03735-6.

D'entrée de jeu, le titre et le sous-titre de cette publication présentent une énigme : l'auteur envisage-t-il de nous présenter la cuisine des premiers arrivants européens de la colonie laurentienne, si l'on se fie au titre, ou viset-il, par un biais détourné, à nous présenter le patrimoine culinaire de la région du Poitou-Charentes?

L'auteur, Vincent Demester, est cuisinier et s'est interrogé sur le patrimoine culinaire québécois suite à un passage à l'Institut de tourisme et d'hôtellerie du Québec. Homme curieux, il s'est intéressé aux traits communs qui unissent le Québec à la région du Poitou-Charentes et a développé une méthodologie pour enquêter sur le patrimoine culinaire, une opération qu'il qualifie de « délicate ».

Après avoir explicité sa méthode, Demester invite son lecteur à passer à l'histoire. Sa première partie, qui fait près du tiers de la publication, porte donc, comme il se doit, sur la région Poitou-Charentes et vise à définir la cuisine de l'Aunis, de la Saintonge, de l'Angoumois et du Poitou. Après avoir esquissé les contours géographiques anciens de ces provinces, l'auteur s'intéresse autant à la peinture ancienne, source malheureusement négligeable pour la région, qu'à la tradition orale telle que dévoilée par la langue et la chanson, avant de s'arrêter au patrimoine matériel des objets de cuisine. Cette démarche lui permet d'aborder le menu populaire de l'Aunis-Poitou : il constate à prime abord l'importance du pain, puis de la soupe, avant d'aborder les sauces - métier oblige sans doute puisque le chef n'hésite pas à mettre de l'avant l'aspect technique relié à celles-ci ! - mais aussi les aliments ichtyens, les viandes, les charcuteries, les légumes et évidemment les desserts.

S'ensuit une esquisse de la cuisine québécoise qui s'articule dans un premier temps autour de la provenance géographique des immigrants du $\mathrm{XVII}^{\mathrm{e}}$ siècle. La démarche tente, tant bien que mal, de dresser un parallèle entre la partie précédente en s'arrêtant à divers aspects de la réalité culinaire 